\title{
Study on the infringement of copyright of micro-blog
}

\author{
Zhou Jianhong \\ College of Humanities and Law \\ Hebei Normal University of Science and Technology \\ Qinhuangdao Hebei, China \\ zhjhong163@163.com
}

\author{
Pei Guanggang \\ Foreign Language College \\ Shandong Normal University \\ Jinan, Shandong, China \\ peigg2005@163.com
}

\begin{abstract}
-micro-blog articles should be protected by Chinese Copyright Law so long as they are original works. The infringement of copyright may be means of copying, digitizing, retweeting works, and setting the hyperlinks in micro-blog. Bloggers need to bear legal liability for their infringement of copyright if knowingly. "Notifying" and "knowing" rules could be applied for micro-blog service provider's legal liability of infringement of copyright without considering the order. It's better for us to build a kind of Internet copyright license contract system in order to solve the problems of copyright authorization in micro-blog
\end{abstract}

Keywords-"Notifying" rules; "Knowing" rules; Authorization contract

\section{INTRODUCTION}

Along with the network popularization and the continuous development of Internet technology, people are increasingly affected by the network. According to statistics, up to the end of December 2012, the number of Chinese netizen reached 564 million; the number of microblog users reached 309 million, there is an increase of 58.73 million from the end of 2011; the percentage of micro-blog users in netizen rose six percent, and reaching to $54.7 \%$. [1]Along with the further promotion of low and mid-range smartphones and increasing users of mobile Internet, micro-blog has been more and more into our life, the number of which is reaching to 202 million, especially up to $65.6 \%$ of twitter users use mobile terminal access to micro-blog, [2]we have entered a micro era. Legal issues, along with micro-blog, however, generated more worthy of our attention. In fact, micro-blog users infringes reputation, privacy and other disputes often arise. However, with the celebrity micro-blog copyright infringement and celebrity micro-blog copyright infringement, micro-blog text copyright infringement problem seems to be a new focus. From the network approach combining technical and legal point of view, I try to explore this problem, with hoping to provide some legislative and judicial reference for our copyright protection on the Internet.

\section{ANALYSIS OF THE EXISTENCE OF COPYRIGHT IN MICRO-BLOG}

On micro-blog, the current controversy bigger issue is whether there is copyright in blog post. Some scholars denied that the blog post with no more than 140 words, is too short to form works, and most content belong to the laundry list, and should not belong to the work. These are restricting the blog itself should not be entitled to copyright. More, the micro-blogs' copyright should not be emphasized for micro-blog has its characteristic of instantaneity and interactive, which making posts spread between bloggers with great randomness. It ought to say that these views have certain rationality. From the origin point of view, micro-blog comes from blog, some of which focus on a particular subject which offers comments or news, most are seen as personal diary, of course, the personal diary is often more casual. And as a kind of micro-blog, micro-blog usually updates within about 140 words, realizing instant share. It is a kind of platform with information sharing, spreading and acquiring, which based on users' relationship. From this perspective, weakening the protection of rights seems to have a certain inevitability.

However, it is inappropriate to judge whether there is micro-blog text copyright issues only according to the number of words or a personal diary of arbitrariness. In terms of number of words, five-character quatrain, which is well-known, has only 20 words. But nobody can deny that their copyrights are worthy of protection, even some people think that one word poem should also enjoy copyright. Although I doubts about this, but we should not deny the possibility of the existence of micro-blog's copyright because of less than 140 words. I thought, the most important thing is to see if the post has the originality to determine whether a post should have the copyright.to determine whether there is copyright in micro-blog, the most fundamental one is to see whether micro-blog is originality or not.

It should be said that micro-blog posts have great randomness and individuality. Some people are willing to remember some laundry list in the post, while others are very attentive to treat the micro-blog, of course, it should not be forgot someone's post containing the content of the above two aspects. To determine whether the works can be protected by copyright law, it has to make a concrete analysis of each post content.

China's implementation of the copyright ordinance provides that "in the copyright law, the term" works "refers to a literary, artistic and scientific domain with original and copy in some tangible form of intellectual property". Undoubtedly, blog posts can be copied, so as long as the works has post original, it can be protected by copyright law in our country. From this point, laundry list of blog posts, even recording a person's personality life, also can not enjoy copyright because of do not have originality. And some of the original posts, which belong to our country works protected by copyright law, are protected. 


\section{PERFORMANCE AND TORT LIABILITY COGNIZANCE OF BLOG INFRINGEMENT OF COPYRIGHT}

Micro-blog posts may constitute works protected by copyright law, however, for others in the blog copyright infringement, not only on blog posts for others' usage of blog posts. In fact, because posts is the micro-blog holders' (bloggers') product of blogging, bloggers' different behavior may directly lead to different infringements of different works. Generally speaking, the blog copyright infringement of performance can be reflected in the following aspects:

\section{A. Directly Copy}

Directly copy means for others' blog posts, is in the micro-blog directly copying others in the micro-blog. This is the most common of the behavior of infringement of copyright in micro-blog. In this case, the micro-blog owner knows he is not the posts' copyright owner, and also copied to others without the prior permission, likely to cause confusion with the copyright owner, it's clearly a infringement of the copyright. because micro-blog holder knows he copied without the copyright, so whether the copy source has the copyright or not, micro-blog holder shall bear tort liability. However, if the replication source have copyright, micro-blog holder post infringes the copyright of the source; if the copy is the infringement of others work, then the micro-blog holder and the copy source belongs to the infringer.

\section{B. $\quad$ The Digital of Others' Work}

This refers to take the others traditional sense of works as his blog posts by means of digital content. This digital process must be done by machine, such as electronic input, digital recording, digital camera, scanner, scanning and so on, its essence is the work of text, graphics, sound, image, value, and converting into a binary encoding process. Some even think that, compared with traditional replication behavior, this behavior is no longer a replication, but more should be a kind of deduction. But it should also see that, the result of this kind behavior, does not produce new works but changing the form of the works. Thus, it is still a copy. In September 1995, the United States launched the "Intellectual Property and the National Information Infrastructure" white paper, which puts this kind of behavior as a reproduction. Through this kind of works' digital, some traditional media such as newspaper' and magazine' work, may be put on to micro-blog. To a certain extent, this kind of way to lift a finger work infringement, is an important part of the copyright infringement. The infringement of copyright shows encroaching on the right of traditional works.

To be sure that, although works' digital also belongs to the form of copy, but it is different from directly copy others in the form of digital works. Because in the form of digital works are often found in Internet, or some storage media, isn't relatively easy to find the real obligee, while traditional forms of works, no matter newspapers, magazines or other publications, are relatively easier to identify the real obligee. Look from here, post directly copy others in the form of digital works and works of traditional forms of digitally to others, the holder of the real level of knowledge is different, so the degree of fault is also different, on the copyright infringement liability, different rules should also be applied.

On tort liability cognizance, the liability cognizance of digitalizing others' work even easier than the responsibility identification of directly copying others' work, because in micro-blog, copies of source of copyright holder is more clearly. Knowing you don't have copyright still put the others works digitally for your blog content, in this case usually don't have to consider other factors to the infringement judgment, micro-blog owner also don't know defend by others enjoy the copyright of.

\section{Setting Up Hyperlinks in Blog post}

For micro-blog, there is another important form, it is to set the hyperlinks in the blog post, which links to other's work, including writing and other audio or video works, it may also be infringement of copyright in others' works. Such as micro-blog bloggers know that other people's blogs or QQ space published infringing works, while they still issued via micro-blog infringing links. So, micro-blog, which sets hyperlink, may also infringe on others' copyright. In practice, many micro-blogs are not directly upload video and music function, but through hyperlinks linking to third-party video or music works. The hyperlink to the third party work behavior is entirely possible infringement of copyright. In fact, because micro-blogs character of social and interactive, hyperlinks in microblogs tend to have quicker than other network behavior mode of transmission, the spread of the faster way is easier to infringement others' works copyright.

For hyperlinks in blog posts, visitors to the micro-blog is not directly visiting posts, but through the hyperlink to access the link works, if the link is the works of the copyright owner of micro-blog holder, it is not infringement; if the link is infringing works, it can do responsibility determination according to the second paragraph of article 36 of the tort liability act, of course if micro-blog's holder intentionally infringing links, microblog's holder may become the infringer together with infringing links managers.

\section{The Forwarding}

Forwarding is one of the most commonly used microblog behavior, to some extent, it is also one of the most common tort behavior. For example, for infringing microblog's forwarding, it can constitute infringement. After forwarding is different from the direct copy, direct copy does not show the replication source, and forwarding can show general source. And the biggest characteristic of micro-blog is highly interactive, forwarding is easy and frequent, and can form outward radiation net-like structure. This kind of common network behavior indeed can also be the most common infringement behavior of copyright.

Forwarding and direct copying others posts are both including the holder of the work, but in forward post forwarding works are marked sources, while in others' work without sources. micro-blog visitors are easy to take direct copy works as original work, and forwarding work doesn't have this kind of problem. So on forwarding and direct copy posts of blog infringement's judgment also should apply different rules.

Forwarding other's work means that makes the forward work of provenance clear, the source may be the work of 
the copyright owner, or may also be infringing works. If forwarding is the copyright owner's work, forwarding behavior belongs to the normal behavior, and is not infringement; If forwarding is the copyrighted works of others, in this case there is possibility of infringement.

All four belong to post directly infringes on the copyright, in addition to this, there is a post of indirect infringement of copyright, that is, intentionally provides the technical measures. This kind of situation mainly means using some technical measures to protect the works, for example, one work was protected by some technical measures, while others post issued directly against technical measures method Although this does not constitute a direct infringement of others works, it belongs to the typical help infringement. In addition, the blog posts link or a hyperlink some web pages of technical measures, may also constitute a infringement of copyright.

For post deliberately provides the technical measures, micro-blog holders takes indirect infringement liability, and because micro-blog holders provide infringing technical measures, so as long as anyone has been using this technical measures to infrings others' copyright, the micro-blog holder should take indirect infringement liability.

\section{TORT LIABILITY COGNIZANCE OF THE MICRO-BLOG SERVICE PLATFORM}

Micro-blog service platform, the micro-blog service provider, is a kind of Internet service provider (ISP). Broadly speaking, network services should include Internet content services, network platform services, network services; in a narrow sense, network services provide services including E-mail, information query, file transfer, BBS and other network services. In fact, with the continuous development of Internet technology, the services provided by the network service provider, become more and more rich and diverse.

Generally speaking, we can analysis the copyright infringement liability of micro-blog service platform from two aspects, one is that micro-blog service platform directly use micro-blog works. In this case, the micro-blog service platform has become a micro-blog's user or manager, so as long as micro-blog post infringes on the copyright, the micro-blog service platform should take the tort liability for copyright owners. This is consistent returns and risk of the principle of requirements. microblog service platform reaps benefits from others' infringement behavior, so it also shall bear the resulting risk, which should be the part of the cost. Secondly, the micro-blog service platform does not use blog content, but it is only a service platform to provide simple information transmission services, this is also the micro-blog service platform as for network service providers' responsibility identification problem. Here I only explore the copyright infringement liability of micro-blog service platform from this aspects.

For the pure copyright infringement liability of information transmission, the United States raised in the network copyright infringement liability limitation act, if only based on the transmission or other reason, provide the online materials contact directly caused tort liability or the vicarious liability of others' infringement behavior. When meet the following conditions, the ISP does not assume tort liability, namely:

- is not the original materials uploaded to the Internet;

- don't production, select, change the contents of the materials;

- does not determine the receiver of materials;

- does not gain benefits from a particular tort;

- does not advertising sponsorship, approval or do advertise;

- does not know or notice or other information so that it is learned that the material is infringing or forbidden by law; unless these required by the ban on the behavior when executed technically feasible and economically rational. Otherwise anyone is not liable.[3] Carry out in the DMCA, when meet the following conditions, the ISP with required transfer function, does not assume tort liability:

- information transmission is provided by others;

- transmission, routing and link, copy process must be achieved through the use of automation technology, and the information without the ISP's choice;

- the ISP can not decide the information receiver;

- the copy formed by the ISP system or network or temporary storage, can be gained by the receiver who scheduled, and usually anyone else can not gain it, and the reasonable time of these copies can't be more than the time required;

- during transmission, information can't have any change in the content.

Judging from China's "tort liability law" regulation, there are two kinds of tort of network service providers for network users assume tort liability, one is the ISP has received users' infringement notice and did not take the necessary measures to stop the expand of the loss, the other one is that the ISP knew users' infringement and did not take measures, in this situation the ISP takes joint and several liability. In addition, as the information transmission of Internet service providers, they do not need to assume tort liability for the user. However, between the two service providers, they may assume tort liability rules, existing quite a lot of controversy, I also tries to put forward some views

\section{A. Notification rules in micro-blog service platform of tort liability cognizance}

China's "tort liability law article 36" in paragraph 2, network users use the web service implementing the infringing act, the patentee shall have the right to notify the network service provider to take necessary steps to delete, block, broken links, etc. Network service provider fails to take necessary measures in time after receiving notice, damage of the expanding part are jointly and severally liable with the network user, this rule is also known to scholars as "notification" rule. This rule should be a reference to the rules of "safe harbor" in the United States.

In China's "Tort Liability Act ", according to the "notice" rule, after receiving notice of infringement of copyright holders, it is necessary for micro-blog service platform to take the necessary measures, or micro-blog service platform should take the responsibility that microblog holders' tort action after being notified. 
However, the "notice" rule relates to an important question, that is for the copyright holder, how to prove their rights and what the extent should be. In this regard, China's "Information Network Transmission Right Protection Ordinance" Article XIV stipulates that to the Internet service providers who provides information on the storage space or provides search and link services, the right holders consider that their services involved in the work, performance, audio and video products, violate their right to network dissemination of information or deleted, changed their electronic rights management information, and then the right holders may submit a written notice to the network service provider, and ask the Internet service providers to remove the works, performances, audio and video products, or cut the links to their works, performances, audio and video products. Of this formal requirements can both protect the the copyright person's rights, but also avoid some of the malicious aggravating the network service provider burden's behavior. This point should be learned from the rules of "Notice \& Take Down" from the United States DMCA. It should be noted that China's "Information Network Dissemination Right Protection Ordinance" provides that copyright person only needs to provide preliminary proof of material without an exact proof material. And for proving the exaction of evidence materials also needs to be judged by the court, which is defined as a preliminary evidence has more reasonable.

In short, for the accountability undertaking after notification of the copyright holder, micro-blog service platform(MSP) only takes the tort liability when failed to take appropriate measures after received the notice, and only takes the tort liability for the infringe liable occur after received the notice.

\section{B. Tort liability cognizance when MSP knew that micro- blog users infringe others' copyright}

Paragraph 3, article 36 of China's "Tort Liability Act" provides that, if the network service provider knows that the Internet users use their network services to violate other's civil rights and interests, while the provider doesn't take necessary measures to restrain this illeagal action, then the provider must bear joint liability with the Internet users. It provides the responsibility that the network service provider should take under the condition that the provider knows that the Internet users infringes the copyright of others. Under this provision, if the micro-blog service platform knew that micro-blog users infringe others copyright, then the micro-blog service platform should bear tort liability, and it is a joint responsibility. This is also the requirements of social control theory. The typical social control theory are control theory and the theory of social contact. Lexus Aires containment theory shows that there are different personal and social attractiveness, which will push and pull to the crime, so it requires people to be self-control and social control to curb individual criminal tendencies.[4] From the point of view of social control, of course, it needs to determine the best controller, in case of micro-blog users' copyright infringement, micro-blog service platform is the best controller, or that the micro-blog service platform control has the lowest cost. Otherwise the infringement micro-blog control costs will be enlarged so that the infringement control becomes more difficult. However, to page 36 of the "Tort Liability Act" , there are two questions need to be mentioned.

1) How to understand the "know" in paragraph 3

For the paragraph 3, Article 36 of "Tort Liability Act", some scholars interpret it as "knowingly" rule, and think that here should be understood as "knowingly"; [5] some scholars believe that here will more accurate to be understood as "known"; [6] and some academics also understand it as "known" rules, and think that here should include that "should have known".[7] And Xi Xiaoming of the Supreme People's Court believes that the "know" should be understood here as "constructive knowledge". [8]After all, how to understand the "know". I think, it can be considered from the following aspects.

From the legislative process, before the "Tort Liability Act" Clause 2, the draft of paragraph 3 of Article 36 has been using the word "knowingly", until the third draft was changed into "know". From this point of view, here can not simply be understood as "knowingly", if it is understood like this, the word "knowingly" can show the meaning clearer, so in the third draft there is no need to change into "know". So, here is not suitable to be understood as "knowingly".

To determine the network service provider's tort liability, it is better to first determine the network service provider's obligations. Once breached the obligation, it shall bear corresponding tort liability. Typically, as a mere transmission of information the network service providers has no obligation to examine the behavior of users, so there is no need for them to tort liability for general users. However, as a network service provider he should at least make a good administrator obligation. For example, microblog service platform users infringe others' copyright. And this incident became the focus of a network, and was brought to the attention of the other newspapers, television and other media. In this case, even if the true micro-blog service platform does not know the user infringement, perhaps there is no reason to give up the tort liability; at least, he violated a network service provider's obligations. And this is an important reason the "know" in paragraph 3 can not simply be understood as "known", but to be understood to include "should have known". In fact, the rule "know" owes much to the U.S. "red flag standards". "Red flag standard" comes from the U.S. Congress DMCA, which takes the user does not know the infringement and the user should be aware that infringement as the conditions that a network service provider take tort liability for the user. According to it, when the network service provider can obviously discover the "red flag" of infringements, if no measures were taken, it will lose eligibility to enjoy the limitation of liability. Red flag standard has both a subjective and objective factors. In determining the network service provider is aware of a red flag, it is necessary to not only take whether the network service provider is aware of the relevant facts and circumstances into account, but also consider whether infringements are already obvious when one in the same or similar circumstances rational. The determination of Red flag criteria is to prevent the perpetrators of "ostrich policy" to evade liability. Although domestic scholars stressed that China's regulations and rules of the DMCA are differ from this, but I think at least the "ostrich" 
enforcer's liability should not be ignored. Here taking the "know" as including "should have known" is obviously more reasonable.

2) The problem of applicable relations in paragraph 2 and 3 Article 36 of "Tort Liability Act"

For the problem of applicable relations in paragraphs 2 and 3, Article 36 of "Tort Liability Act", some scholars believe that paragraph 2 is generally applicable terms and paragraph 3 is only applied in exceptional conditions. Some scholars believe that paragraphs 2 and 3 are juxtaposed terms, the right holder may choose to obey. I think that the discussion of this issue may go back to the legal relationship in the past, the contents of the legal relationship is nothing more than rights and obligations. And the acceptance of liability is arising based on the legal obligations from non-performance. First, when rights holders have received a notice of infringement, he shall take appropriate measures to avoid the loss of rights continuing to expand. Second, although the rights holders have not received a notice, when knowing infringed by others, he should also take appropriate measures. But as a good administrator, he should also avoid becoming inaction "ostrich policy" enforcer. Violating to these three legal obligations constitutes paragraphs 2 and 3.

\section{Other micro-blog service platforms' copyright infringement}

When refer to micro-blog service platforms' copyright infringement, it should also consider a situation, that is, those micro-blog service platforms have not directly service relationship with the micro-blog content. Unauthorized micro-blog service platform published blog context on behalf of other person registered on other platform, which is clearly contrary to the wishes of registered holders of the micro-blog platform, also infringes on other micro-blog service platform rights.And these micro-blog holders that false by other platforms mostly are celebrities. They have a strong fans or followers, and their micro-blog content are protected by copyright law works.For micro-blog using, regardless of whether they are used for direct commercial; it will give some economic benefits to other platform. Then its use may infringe copyright in works micro-blog. From this point of view, as long as other micro-blog service platforms post bogus content under others' name; then they shall assume responsibility for copyright infringement.

In addition, there are many other bodies on the network, such as BBS service platform, mail service platforms and other network services platform. They are likely to involve micro-blog content reprint issues and related to copyright infringement, some of the traditional media, such as newspapers, magazines, may also become the subject of copyright infringement. When they use micro-blog content, if they do not provide the source, may also become the subject of copyright infringement, and then they shall bear tort liability.

\section{Establishment of authorization contract of post copyright}

It is beyond question that microblog copyright needs to be protected, and there is also a basic path for copyright infringement liability maintaining. However, the degree of protection of copyright microblog content is an elusive subject. If there was too little protection, it can affect the enthusiasm of artists and the development of science and technology; if there was too much protection, it will affect the dissemination of works. For a national science and technology and cultural development is not a good thing, even for the development of the Internet itself may also have a negative impact, thus restricting the development of network application itself. According to balance theory of intellectual property rights, copyright laws should seek a new balance between the protection of copyright and the promotion of culture, science and art development; and the promotion of network technology development and application. This needs to require copyright protection for microblog content, at the same time take responsibility for copyright infringement then have certain restrictions.

In fact, the sharing of resources is the basis for existence and development of the Internet, the emergence and development of the Internet. It is precisely to allow more people get more shared resources more quickly and easily. This feature also determines the openness of the Internet itself, so that all the information on the Internet by default to all who are open to the public. Of course, since everyone expected for their right to information differently, and therefore, it should also allow the owner of information to customize the information openness. According to contract theory proposed by Japanese scholars Kitagawa Zentaro, there is a kind of information on the Internet as a transaction object covenants.[9] Domestic scholars have even suggested that there should establish an Internet copyright license contract. And the contract's scope of authority should be expressed or implied by the scope prevail of the copyright owner's behavior. When using others' works, every internet user can not change the use of the works of others copyright owner, it can not work against the purpose of the copyright holder. [10]Accordingly, let us examine the microblog. as a kind of sociability platform for information sharing and dissemination, the mocroblog is produced to meet the needs of a social and interactive. So the normal share and flow of microblog content and information should not be restricted. As long as it is not contrary to the author's own behavior, it can presume the scope of authorization based on their microblog behavior. Of course, it can not deny the protection of personal rights in copyright, and otherwise it may deny the author's copyright. According to microblog copyright contracts system of microblog content, we can have a clearer regulation on different subjects.

For microblogging holder, he is the direct controller of microblog content, who know best whether their contents are original or not. If it is from the works on the network, and belongs to others published works and unrestricted, you can fully reproduced without the consent of the author. Of course, at the same time it should be sure to indicate the source reproduced. If the content belongs to other people' s published work and imposes certain restrictions or afford a certain use conditions, microblog holders can't change the protection of others' works, even reproduced without permission. If it is someone else's works published through traditional media, and the author did not authorize release on the network, you can not upload the works of others online and post infringing link through microblog without permission. 
For the microblog platform, according to Microblog Registration User Agreement, it is without further discussion according to the contract, to use any blogger's original works, including commercial and non-commercial use. If non- original works, there may be the risk of infringement, which is consistent with gains - risk consistent theory. The identification of specific responsibilities can be implemented under the Article 36 of "Tort Liability Act". It is worth mentioning that when it comes to the "notice" rule, the microblog service platform enjoy a range of defenses, such as Article XIV of "Information Network Transmission Right Protection Ordinance", when noticing, the copyright owner requirements provide prima facie evidence of the copyright, otherwise microblog service platform can refuse to remove the relevant content or disconnect the link.

And according to Article XVI of "Information Network Transmission Right Protection Ordinance", after service objects received the notice transferred to the network service provider, they think that their providing works, performances, audio and video products do not infringe the rights of others, they can submit a written explanation to network service providers for the restoration of the deleted works, performances, audio and video products, and being disconnected or restore works, performances, audio and video products link. In other words, in the application of "notice" rule, the microblog platform can also not remove any related contents, or restore broken links under the requirements of users. Of course, microblog users also need to provide proof of their initial rights.

For the identification of other microblog service platforms copyright infringement, cannot be contrary to the works' authorization of microblog holders. From the microblog users' aspect, each user can reproduced others microblog contents cross-platform, as long as there is a clear provenance. But for the micro-blogging service platform, it should respect the choice of the microblog holder. Therefore, when using other works registered in other service platforms, the microblog service platform must be agreed by the author, the kind of restrictions of registration platform are not entitled to the platform that have not registered. In addition, for the use of microblog contents by other BBS platform, messaging platforms, they are often dominated by user. And the use of work by these platforms users should be free to reproduce, which is allowed by Internet copyright authorization contract, if he can identify the source of information without infringing of copyright. But if other platforms microblog users infringe copyright in works, then BBS platform or e-mail platforms should take the tort liability based on "notice and delete" rules, which is the responsibility of the other platforms bodies, also the limitation of its liability for infringement of copyright.

\section{CONCLUSION}

Nowadays, the Internet has penetrated into our study, work and life. And promote scientific, artistic and cultural dissemination of works and protection of interests of copyright holders is that we must face two problems simultaneously. Obviously, this is a contradiction itself, but it is difficult to make an either-or choice. Because of the continuous development of the Internet, it makes the science, culture and art works' dissemination more convenient and faster. This new way of communication enables people around the world to share the latest work in the field of science, culture and art in the shortest amount of time. And the content of copyright rights and the ways to exercise rights has been greatly enriched. On the other hand, the rights of copyright holders are increasingly vulnerable to abuse. How to balance and coordinate the dissemination of science, culture, communication and artistic works and the protection of rights of the copyright owner is the problem to be solved today. Also, solutions to such problems cannot fundamentally solve the damage of the Internet as well as the development of networking technology. The protection of copyright in micro-blog content is also an important embodiment of contradictions.

The infringement problem of mocroblog content copyright seems to be an issue to protect the rights in micro works. But this right can not be ignored as a micro right. In order to promote the dissemination of scientific and cultural works and the application and development of network technology, also to protect the enthusiasm of creators, it is necessary for us to bring attention to microblog when we enjoy micro-blog culture, but also to give an appropriate protection to micro-blog copyright.

\section{REFERENCES}

[1] Statistical Report on Internet Development in China, 31 [R].China, China Internet Network Information Center . 2013.1

[2] Li Wei. Discuss on Copyright and Supervision on Microblog[J]. Chinese Broadcast, 2012,4: 7-11

[3] U.S. Copyright Office Summary. The Digital Millennium Copyright Act of 1998[EB/OL]. United States. 2013.12.30.http://www.copyright.gov/legislation/dmca.pdf

[4] Wu zongxian.History of Western Criminology [M]. Lawman Education Press. 1997

[5] Wang Liming. Paraphrase of the Tort Liability Act[M].2010

[6] Yang Lixin. Comprehend and Interpretation on Internet Tort Liability about the Tort Liability Act[J]. Academic Journal of National Prosecutor College of PRC. 2010.2: 3-10

[7] Yuan Xueshi, Chen Yi. Study on the third Paragraph of Article 36 of the Tort Liability[J]. Electronics Intellectual Property.2012.2: 3-10

[8] Xi Xiaoming. Comprehend and Application of Articles of the Tort Liability Act[M].People' Court Press.2010

[9] (Japan) Kitagawa Zentaro.Information in Internet , Copyright, License Contract[J].(Ju Tao Translate) Global Law Review, 1998,3:39

[10] Zhou Jianhong, Yang Xiaolin, Discuss on License Contract \& fair use of Copyright on Internet[J]. Journal of Hebei Normal University of Science \& Technology:2007.3:60-63 